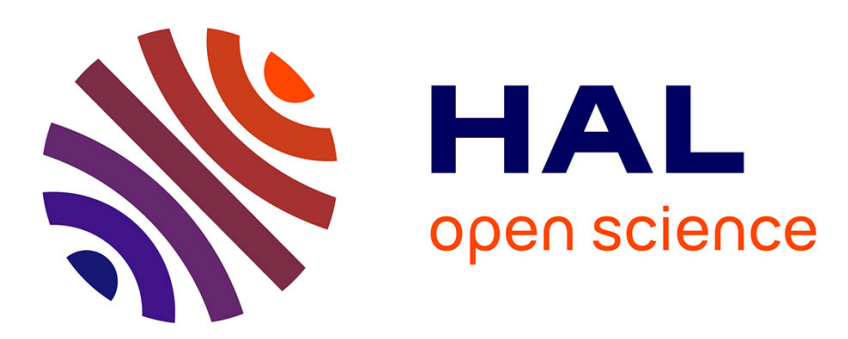

\title{
A signal processing method: Detection of Lévy flights in chaotic trajectories
}

\author{
Francoise Briolle, Benjamin Ricaud, Xavier Leoncini
}

\section{To cite this version:}

Francoise Briolle, Benjamin Ricaud, Xavier Leoncini. A signal processing method: Detection of Lévy flights in chaotic trajectories. Nonlinear Science and Complexity (NSC), 2012 IEEE 4th International Conference, Aug 2012, Budapest, Hungary. pp.19-24, 10.1109/NSC.2012.6304726 . hal-00963779

\section{HAL Id: hal-00963779 \\ https://hal.science/hal-00963779}

Submitted on 22 Apr 2014

HAL is a multi-disciplinary open access archive for the deposit and dissemination of scientific research documents, whether they are published or not. The documents may come from teaching and research institutions in France or abroad, or from public or private research centers.
L'archive ouverte pluridisciplinaire HAL, est destinée au dépôt et à la diffusion de documents scientifiques de niveau recherche, publiés ou non, émanant des établissements d'enseignement et de recherche français ou étrangers, des laboratoires publics ou privés. 


\title{
A signal processing method : detection of Lévy flights in chaotic trajectories
}

\author{
Francoise Briolle ${ }^{*}$, Benjamin Ricaud ${ }^{\dagger}$, Xavier Leoncini * \\ * Aix Marseille Université, Université de Toulon, CNRS, CPT, UMR7332, 13288 Marseille, France \\ Email: briolle@cpt.univ-mrs.fr, xavier.leoncini@cpt.univ-mrs.fr \\ ${ }^{\dagger}$ Lab. Analyse, Topologie, Probabilité, Aix-Marseille Université, France \\ Email: bricaud@cmi.univ-mrs.fr
}

\begin{abstract}
Transport in low dimensional Hamiltonian chaos can be anomalous due to stickiness and rise of Lévy flights. We suggest a signal processing method to detect these flights in signals, in order to characterize the nature of transport (diffusive or anomalous). We use time-frequency techniques such as Fractional Fourier transform and matching pursuit in order to be robust to noise. The method is tested on data obtained from chaotic advection.
\end{abstract}

\section{INTRODUCTION}

Characterizing anomalous transport in low dimensional Hamiltonian systems and quantifying its impact is of crucial importance in different fields of physics. One can for instance think of mixing related problems in oceans or atmospheres, or in micro-fluidic devices, but also in magnetically confined fusion plasmas etc...[1], [2], [3], [4], [5], [6], [7], [8], [9] In order to analyze chaotic transport, several tools are being used such as a fractal analysis of the trajectories, giving Lyapunov exponents, multi-fractal analysis. In this article, we suggest a method for analyzing anomalous transport, when it is dominated by intermittent behavior and long lasting Lévy flights. In this situation, the individual particle motion displays typically periods of ballistic transport (Lévy flights) in between chaotic motion which looks like standard random walks (Brownian motion). From a statistical point of view, this can generate anomalous transport phenomenon and this anomaly can be quantified for instance by measuring the characteristic exponent of the variance growth. We may also try to quantify it with more details and try to characterize in a more accurate way by detecting and counting the amount and duration of these Lévy flights. For this purpose it is important to remember that in most experimental data noise is present in the signal to analyze. This noise may impact the classical statistical methods used to quantify transport. At variance, our signal processing analysis is able to detect and extract levy flights even if embedded in noise (of reasonable amplitude). This is definitively a plus when considering using the method in practical situations.

To be more precise, the signal processing method relies on the so-called uncertainty principle. This principle simply states that time and frequency (or momentum and position in quantum mechanics) can not be known simultaneously with arbitrary precision: If $\Delta t$ is the accuracy of the measure in time and $\Delta f$ is the accuracy in frequency, the Heisenberg principle implies that:

$$
\Delta t \cdot \Delta f \geq c,
$$

where $c$ is a strictly positive constant. This phenomenon is usually seen as a problem and many works have been focusing on trying to minimize the uncertainty $c$. Conversely, we shall take advantage of it. Indeed, anticipating on the precise description of our method, we transfer the tracer trajectory seen as a signal into the time-frequency plane. As a direct consequence of the uncertainty principle the Brownian motion becomes blurry while the ballistic flights remain distinct. Thus, the detection of Lévy flights becomes the detection of straight lines in the time-frequency plane. This can be efficiently done by a projection onto a family of bases chirp functions [10] and in the end, the uncertainty principle gives us the ability to accurately detect flights even in the presence of noise. Considering the numerical aspects, it is also important to note that this procedure is fast and relying on the fast Fourier transform: the complexity is of $\mathcal{O}\left(N^{2} \log N\right)$, where $N$ is the size of the sampled data.

This paper is organized as follows, first, in part II we present a special physical problem, namely the phenomenon of chatic advection, which motivated the establishment of our signal processing technique and will provide us the data to test it with. The notion of transport and Lévy flight is as well stated with precision along with the type of data to analyze. Then, in part III the method for analyzing this particular anomalous transport is presented. The method is then tested using data from part II. In fine, the results of the detection of Lévy flights are presented.

\section{Chaotic ADVECTiOn PHENOMENA}

In this section we discuss the stickiness that occurs in low-dimensional Hamiltonian systems. To bemore specific, we consider more specifically the phenomenon in the setting of chaotic advection of passive tracers in a flow generated by three vortices.

\section{A. Definitions}

We first briefly describe the advection phenomenon. For this purpose, we consider the flow $\mathbf{v}(\mathbf{r}, t)$ of an incompressible 
fluid $(\nabla \cdot \mathbf{v}=0)$. In this setting the trajectories of passive particles $^{1}$ are solutions of the following differential equation:

$$
\dot{\mathbf{r}}=\mathbf{v}(\mathbf{r}, t),
$$

where $\mathbf{r}=(x, y, z)$ corresponds to the passive particle position. When the flow is two-dimensional, the motion becomes Hamiltonian, indeed since $\nabla \cdot \mathbf{v}=0$, we defined a stream function $\boldsymbol{\Psi}$ such that $\mathbf{v}=\nabla \wedge \boldsymbol{\Psi}$, and for two-dimensional flow, $\Psi=\Psi \mathrm{z}$ corresponds actually to a scalar field $\Psi$. We can then rewrite Eq. (1):

$$
\dot{x}=\frac{\partial \Psi}{\partial y}, \quad \dot{y}=-\frac{\partial \Psi}{\partial x} .
$$

A peculiar feature of this Hamiltonian is that the physical space is identified to the phase space as $x$ and $y$ are canonical conjugate variables of the Hamiltonian $\Psi$. Note that we obtain a one dimensional integrable Hamiltonian system if $\Psi$ is independent of time, which just means that that particles follow stream lines. If $\Psi$ depends on time, we generically obtain Hamiltonian chaos and a system with $1-\frac{1}{2}$ degree of freedom. This chaotic nature of the trajectories is in this context referred to the phenomenon of Chaotic advection: even if the flow has a laminar (non turbulent) structure, passive particles or tracers display Hamiltonian chaos[11], [12], [13]. Becasue of this phenomenon, mixing is considerably enhanced in chaotic regions, as usually the erratic motion due to chaos motions mixes much faster than the microscopic molecular diffusion [14], [15], [16]. This phenomenon becomee the method of choice when mixing fragile molecules in micro-fluidic devices. There are also a multitude of physical systems and applications as for instance in geophysical flows or magnetized fusion plasmas [1], [2], [3], [4], [5], [6], [7], [8], [9].

To generate the flows from which we will analyze the data given by trajectories, we consider a flow generated by a system with three point vortices. Before moving on, we briefly describe what a system of point vortices is.

\section{B. A system of point vortices}

We shall start with the Euler equation, which for the vorticity in a two-dimensional incompressible flow writes:

$$
\frac{\partial \Omega}{\partial t}+[\Omega, \Psi]=0, \Omega=-\nabla^{2} \Psi
$$

where $[\cdot, \cdot]$ and denotes the Poisson brackets. If we now consider a vorticity field given by a superposition of Dirac functions:

$$
\Omega(\mathbf{r}, t)=\sum_{i=1}^{N} k_{i} \delta\left(\mathbf{r}-\mathbf{r}_{i}(t)\right)
$$

where, $k_{i}$ designate the vorticity of the point vortex localized at point $\mathbf{r}_{i}(t)$; we find that this so-called point vortex distribution is a solution of the Euler equation if the vortices have a specific dynamics [17]. To be more precise, vortex motion results from

\footnotetext{
${ }^{1}$ also referred as tracers
}

$N$-body Hamiltonian dynamics whose Hamiltonian writes (on an infinite plane):

$$
H=\frac{1}{2 \pi} \sum_{i>j} k_{i} k_{j} \ln \left|\mathbf{r}_{i}-\mathbf{r}_{j}\right|,
$$

where and similarly to the passive tracers $k_{i} y_{i}$ and $x_{i}$ are the canonically conjugate variables of the Hamiltonian (5).
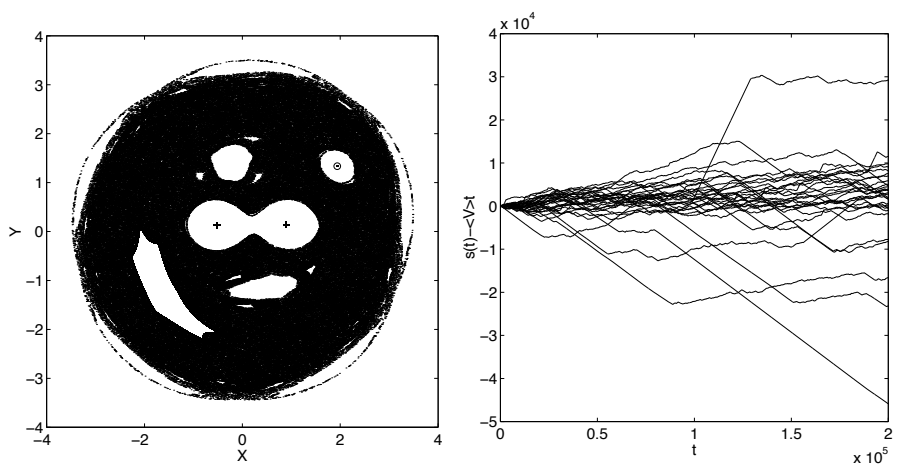

Fig. 1. Left: Poincaré section of a system of three point vortices. Vorticities are $(-0.2,1,1)$. Right: Deviation from average arc-length $(s(t)-V t)$ versus time for an ensemble of 30 particles advected in the flow. We notice the presence of Lvy flights. The run is performed over 20000 (quasi-)periods of the vortex motion.

We obtain as well the stream function, namely the Hamiltonian of passive tracers:

$$
\Psi(\mathbf{r}, t)=-\frac{1}{2 \pi} \sum_{i=1}^{N} k_{i} \ln \left|\mathbf{r}-\mathbf{r}_{i}(t)\right| .
$$

We shall now make a few remarks regarding the Hamiltonian (5); it is invariant by translation and by rotation in the plane, giving rise to two extra independant constant of the motion besides the energy. It can then be shown the system is integrable if the number of point vortices $N$ is such that $N \leq 3$, on the other hand vortex motion is not integrable and consequently chaotic if $N>3$ [18], [19]. To mimic a laminar flow, we consider a regular one time dependent one, and this we consider a the flow generated by three vortices. Also, and since we are interested in asymptotic transport properties we have considered initial conditions giving rise to a periodic motion of the vortices. We now briefly discuss the transport properties of advected particles.

\section{Stickiness and anomalous transport}

Before considering the system of point vortices per se let us precise the nomenclature, that we shall consider to characterize transport. In fact the classification of the type of transport is usually based on the value of the characteristic exponent of the evolution of the second moment.

Transport is said to be anomalous if it is not diffusive in the sense $\left\langle X^{2}\right\rangle \sim t^{\mu}, \mu \neq 1$

1) If $\mu<1$ transport is anomalous and one refers to it as sub-diffusion

2) If $\mu=1$ transport is Gaussian and one refers to it as diffusion 
3) If $\mu>1$ transport is anomalous and one refers to it as super-diffusion.

In fact to be more precise we should consider all moments of the distributions and not only the variance, and this can lead to more sublte refinenemnt in the different type of transport properties (self-similar, multifractal etc...) .

For the specific case of the advection in the flow of three point vortices depicted in Fig. 1, we have to specifiy what transport properties we are considering. Indeed one notices a chaotic sea filled with isalnds of stability, so the system per se is not ergodic (in the sense that there are no trajectory that becomes as close as possible of any phase space point). So the transport is considered for trajectories in teh choatic sea. However since this chaotic area is bounded, using positions to measure dispersion is not usually not a good idea, as teh boundaries are usually quickly reached and it becomes difficult to extract a characteristic exponent on such short times. In order to circumvent this problem, we monitor "transport properties" of the lengths of trajectories, actually we monitor the dispersion among different initial conditions of this quantity

$$
s_{i}(t)=\int_{0}^{t}\left|v_{i}(\tau)\right| d \tau,
$$

where $v_{i}(\tau)$ is the norm of the speed (in phase space, but identical to the real spead) of particle $i$ at time $\tau$. Then we compute the moments

$$
M_{q}(t) \equiv\left\langle|s(t)-\langle s(t)\rangle|^{q}\right\rangle,
$$

where $\langle\ldots\rangle$ corresponds to ensemble averaging over different trajectories and then we extract a characteristic exponent from the evolution of the different moments

$$
M_{q}(t) \sim t^{\mu(q)} .
$$

It was shown that for the point vortex flow, the transport is superdiffusive and multi-fractal [20]. These anomalous features were traced back to the phenomenon of stickiness: when a trajectory arrives in the neighbourhood of an island of stability it can get stuck around the island for arbitrary large times which act as pseudo-traps, and displays therefore a Lévy flight in the evolution of the length of teh trajectory. This generates strong memory effects (slow decay of correlations) and as a consequence displays anomalous transport properties. We have drawn in Fig. 1 the relative evolution of the length with respect to the mean of an ensemble of 30 different particles. And indeed can see that the time evolution is reminiscent of some random walks by parts coming from the chaotic sea and there are some parts where the evolution looks regular and ballistic usually referred to as Levy flights, each different slope corresponding to a different sticky region.

\section{TIME-FREQUENCY METHOD}

We shall now introduce the particularities of the data set from a signal processing point of view and describe the analyzing method. For clarity, the result of each step will be illustrated with applications to the simulated data of the previous part (trajectories of tracers evolving in the flow generated by three vortices).

\section{A. The data set}

From a typical arc-length trajectory it is possible to get a one-dimensional signal $m(t)$ of $N=1000$ sampling points, $t \in[1, N]$. A set of signals is shown on Fig. 1 (right), and one of them on Fig. 2 (left). Several parts can be distinguished: a random fluctuation (Brownian motion) and some almost linear segments of different length corresponding to Lévy flights. Our method is dedicated to the detection of these linear parts and the estimation of their length and slope (proportional to the velocity $\Delta h / \Delta t)$.

\section{B. The detection method}

As illustrated in Fig. 1 (right) and Fig. 2 (left), Lévy flights correspond to an almost linear evolution of the arc length. It is then important to notice that due to the uncertainty principle:

- random fluctuations in frequency cannot be rendered precisely in the time-frequency plane. It requires to be precise both in time and frequency, which is forbidden.

- linear parts or more generally slowly varying frequency components are emphasized by the time-frequency representation. Moreover, linear parts, called chirp signals, can be detected efficiently using the fractional Fourier transform.

It is hence interesting and natural for us to take advantage of this fact for the analysis of the data set. To perform our analysis we shall therefore considered $m(t)$ as the phase derivative of a new signal $M(t)$. This corresponds to the first step of the process: let us introduce the phase

$$
\begin{gathered}
\varphi(t)=\int_{1}^{t} m(\tau) d \tau \\
M(t)=e^{i \varphi(t)}=e^{i \int_{1}^{t} m(\tau) d \tau} .
\end{gathered}
$$
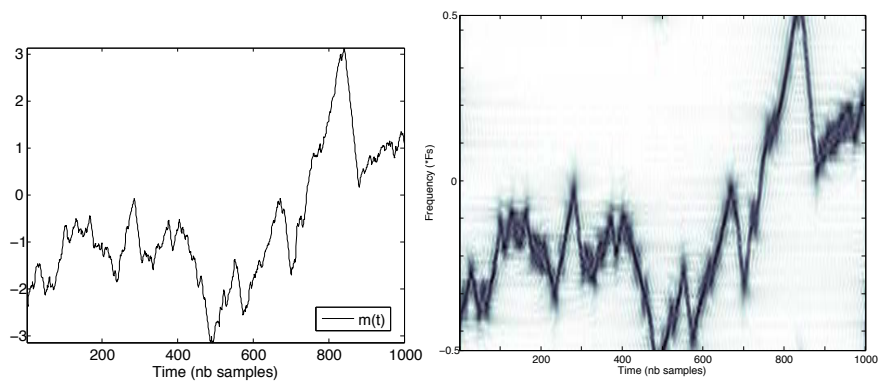

Fig. 2. left: time representation of $m(t)$, arc-length of a single particle. right: short-time Fourier transform representation of the signal $M(t)$.

The above signal $M(t)$ is a non-stationary signal of magnitude one and the phase derivative (instantaneous frequency) $f_{M}(t)$ is equal to $m(t)$ :

$$
f_{M}(t)=\frac{d \varphi(t)}{d t}=m(t)
$$


The time-frequency representation of $M$ on Fig. 2 (right), also called the spectrogram[21], shows the fluctuations of the phase derivative $f(t)$ as a function of time. This frequency component mimicks the behavior of the signal $m$, but the important difference is that, thanks to the uncertainty principle, Brownian fluctuations become diffuse stains (see Fig. 2 (right)). As a consequence pure random behavior is blurred, but linear parts remain sharp. Our first objective is attained: the linear behavior has been emphasized over the Brownian motion.

We now move on to the second part of the process. We are looking for lines in the time-frequency 'picture'. For this purpose we project the signal $M(t)$ on a family of orthogonal basis of chirps signals:

- Given a parameter $\theta_{0} \in(-\pi, \pi)$, we introduce the basis of chirps $\left\{\psi_{\theta_{0}, \mu}\right\}_{\mu}$ with a frequency slope of $\frac{1}{\tan \theta_{0}}$,

$$
\psi_{\theta_{0}, \mu}(t)=e^{i\left(\frac{1}{2 \tan \theta_{0}} t^{2}+\frac{\mu}{\sin \theta_{0}} t\right)} .
$$

Since $t \in[1, N], \mu=2 \pi n / N$ with $n \in[1, N]$. The phase derivative (instantaneous frequency) of $\psi_{\theta_{0}, \mu}(t)$ is equal to :

$$
f_{\psi}(t)=\frac{1}{\tan \theta_{0}} t+\frac{\mu}{\sin \theta_{0}} .
$$

Notice that $\mu / \sin \theta_{0}$ is the frequency value at $t=0$ (frequency offset) of the chirp $\psi_{\theta_{0}, \mu}$. The projection of the signal is described by the following procedure:

$$
C\left(\theta_{0}, \mu\right)=\sum_{t=1}^{N} M(t) \overline{\psi_{\theta_{0}, \mu}(t)},
$$

where the bar denotes the complex conjugate.

This projection is equivalent to the fractional Fourier transform (up to a normalizing factor) [22].

Due to the orthogonality of the basis $\left\{\psi_{\theta_{0}, \mu}\right\}_{\mu}$, it is possible, from the projection, to re-synthetize the signal $M(t)$ :

$$
M(t)=\sum_{\mu} C\left(\theta_{0}, \mu\right) \psi_{\theta_{0}, \mu}(t),
$$

- Since Lévy flights may have different slopes, it is necessary to project the signal onto a set of $\mathrm{P}$ bases of chirps $\left\{\psi_{\theta_{i}, \mu}\right\}_{\mu}$, with $\mathrm{P}$ values of $\theta_{i} \in(-\pi, \pi)$. We get a $P \times N$ matrix $C\left(\theta_{i}, \mu_{j}\right)$ of projections.

- When the characteristics of a chirp (frequency slope and offset) match the one of a "frequential picture", $\left|C\left(\theta_{i}, \mu_{j}\right)\right|$ is a maximum.

Taking the signal $m(t)$ shown in Fig. 2 as an example, there is a specific direction $\theta_{n}$ (related to the slope of the largest Levy flight) where several maxima could be detected. Using a threshold, four main maxima are localized $\left|C\left(\theta_{n}, \mu_{1}\right)\right|,\left|C\left(\theta_{n}, \mu_{2}\right)\right|,\left|C\left(\theta_{n}, \mu_{3}\right)\right|,\left|C\left(\theta_{n}, \mu_{4}\right)\right|$ for $\mu_{1} \sim 400, \mu_{2} \sim 750, \mu_{3} \sim 780, \mu_{4} \sim 850$ (dash lines on Fig. 3).This give evidence that there are four Lévy flights with a particular slope $\frac{1}{\tan \theta_{n}}$. This process detects linear parts in the time-frequency plane.

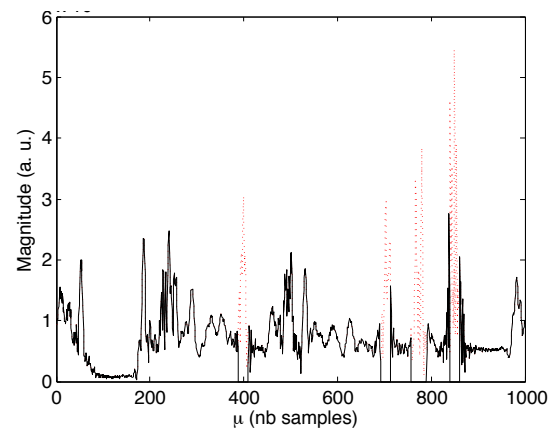

Fig. 3. For $\theta_{n}$, signal projections $\left|C\left(\theta_{n}, \mu\right)\right|$.

The detected Lévy flights can be extracted from the original signal $M(t)$ thanks to the orthogonal projection. They can be gathered in a new signal $M_{1}(t)$ :

$$
M_{1}(t)=e^{i \frac{1}{2 \tan \theta_{n}} t^{2}} \sum_{\mu_{1}, \mu_{2}, \mu_{3}, \mu_{4}} C\left(\theta_{n}, \mu\right) e^{i \frac{\mu}{\sin \theta_{n}} t},
$$

which may be used later for futher investigations on these slopes (future work). This step is illustrated in Fig. 4 (left), which represents the short-time Fourier transform of the newly recreated signal $M_{1}$. The four main Lévy flights are visible as four dark lines. Notice that since it is an orthogonal projection, one can write:

$$
M(t)=M_{1}(t)+M_{1}^{\perp}(t),
$$

where $M_{1}^{\perp}$ contains the rest, i.e. the Brownian motion and (possibly) shorter lévy flights. As it is shown on Fig. 4, the Lévy flights (right) velocities and durations are related to the caracteristics of the extracted components (right): The velocity is propotional to the chirp slope $\frac{1}{\tan \theta_{n}}$, the duration to the chirp length.
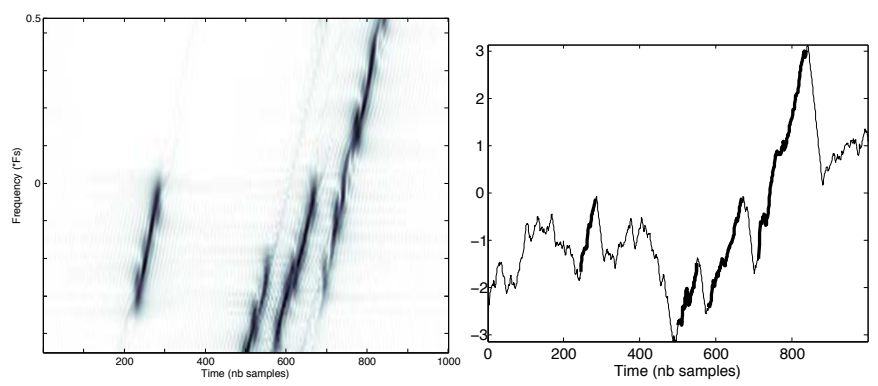

Fig. 4. Left: Short-time Fourier transform of the signal $M_{1}$; four Lévy flights have been detected. Right : Comparison of signals $m(t)$ and $m_{1}(t)$; characterization of Lévy flignts.

To detect secondary flights, the above procedure may be iterated but this time on $M_{1}^{\perp}(t)$ until all the flights larger than a predefined length are detected. This is the principe of the matching pursuit [24]. It allow to obtain a final rest $M_{f}$ where only Brownian noise is present. This open the way to the analysis of the residual noise as the flights have all been efficiently extracted (ongoing work). This should be of great interest when analysing experimental signals. A faster but 
slightly less accurate analysis may be done by picking directly all the maxima of the matrix $C$, avoiding the iterative scheme. Extracting all the flight at once may create small artifacts, inducing small errors in the calculus of flight durations; the matching pursuit has been designed to avoid such artifacts.

The steps of the process can be summarized as follow, for a single trajectory :

- Trajectory $m(t)$ as a phase derivative of a signal $M(t)$ : time-frequency transformation

- Search for lines in the time-frequency 'picture' : projection on a basis of chirps

- Lévy flight detection : peak picking on the matrix $C$ and partial synthesis of $M_{1}(t)$ (with or without matching pursuit)

- Characterization of the Lévy flights.

Remark 1: The computational complexity for obtaining the matrix $C$ is of order $N^{2} \log N$. For each $\theta$ the projection onto the chirp basis is performed via a fast Fourier transform[23], [22] of complexity $N \log N$. This is done for a number of $\theta$ proportional to $N$.

Remark 2: For Lévy flights with steep slopes, numerical problems may arise due to the discretization. The solution used here is to make a 90 degrees rotation of the signal in the time-frequency plane before the projection on chirps and adapt the values of $\theta$ in consequence: this rotation is simply obtained by applying a Fourier transform to the signal $M$.

\section{REsults}

As a test of the method we now consider the data obtained from the advection of 253 tracers in the point vortex flow described in section 1. Our goal is to detect the multi-fractal nature of the transport resulting from the sticky islands, which would serve as a proof of concept and pave the way to apply the method to numerical and experimental data.

In [20] transport was analyze with traditional tools and found to be anomalous and super diffusive. The origine of the anomaly was trace back to a multi fractal nature of transport linked to stickiness on four different regular regions. One would thus expect four different type of Lévy flights in the data.

The method was applied on the data. We localize Lévy flights and meas. The results are displayed in Fig. 5, where duration is the function of velocity. As it can be seen, four different type of flights are detected, and their corresponding velocities are in good agreement with the result found in [20], confirming the accuracy of the analyzing signal processing method.

\section{CONCLUSIONS}

The first step of the method help emphasing the straight lines over random fluctuations.

The second step consists in the detection of straight lines in the time-frequency image. The Fractional Fourier transform applied to a one-variable signal is similar to a Radon transform or Hough transform of a standard image.

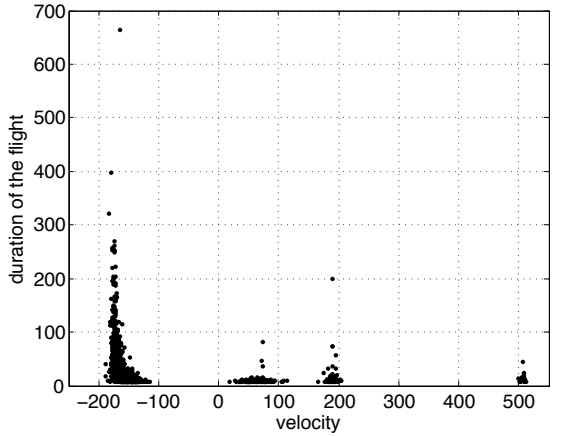

Fig. 5. For 253 arc-length trajectories, lengths of the Lévy flights as a function of the velocity $(\Delta h / \Delta t)$. Four main distributions of the velocity can be observed.

This method and its first results open the way to more systematic detections of Levy flights in anomalous transport phenomena. The detection algorithm is efficient and fast, allowing the analysis of a large number of tracers trajectories in a short time. The output, yielding the number Levy flight and their duration, can be analysed in a second step by statistical tools (e.g. mean number of flight in a trajectory, mean length, variance,...). This will lead to a more accurate characterization of this particular case of anomalous transport.

\section{Open Questions}

- Can we analyse the remaining random signal $S_{n}$ and recover Brownian motion?

- This method can detect noisy flights, what is the maximal level of noise admitted?

- What is the minimal length of a Levy flight?

- Is it possible to quantify anomalous transport with this technique?

\section{REFERENCES}

[1] M. Brown and K. Smith, Phys. Fluids 3, 1186 (1991)

[2] R. P. Behringer, S. Meyers and H. L. Swinney, Phys. Fluids A 3, 1243 (1991).

[3] A. A. Chernikov, B. A. Petrovichev, A. V. Rogal'sky, R. Z. Sagdeev and G. M. Zaslavsky, Phys. Lett. A 144, 127 (1990).

[4] F. Dupont, R. I. McLachlan and V. Zeitlin, Phys. Fluids 10, 3185 (1998).

[5] A. Crisanti, M. Falcioni, A. Provenzale, P. Tanga and A. Vulpiani, Phys. Fluids A 4, 1805 (1992).

[6] B. A. Carreras, V. E. Lynch, L. Garcia, M. Edelman and G. M. Zaslavsky, Chaos 13, 1175 (2003).

[7] S. V. Annibaldi, G. Manfredi, R. O. Dendy and L. O. Drury, Plasma Phys. Control. Fusion 42, L13 (2000).

[8] D. del Castillo-Negrete, B. A. Carreras and V. E. Lynch, Phys. Plasmas 11, 3854 (2004).

[9] X. Leoncini, O. Agullo, S. Benkadda and G. M. Zaslavsky, Phys. Rev. E 72, p. 026218 (2005).

[10] F. Briolle, R. Lima, V.I. Man'ko and R. Vilela MendesMeas. Sci. Technol. 20, 10, (2009)

[11] H. Aref, J. Fluid Mech. 143, 1 (1984).

[12] H. Aref, Phil. Trans. R. Soc. London A 333, 273 (1990).

[13] J. M. Ottino, Ann. Rev. Fluid Mech. 22, 207 (1990).

[14] J. M. Ottino, The Kinematics of mixing: streching, chaos, and transport (Cambridge U.P., Cambridge, 1989).

[15] G. M. Zaslavsky, R. Z. Sagdeev, D. A. Usikov and A. A. Chernikov, Weak Chaos and Quasiregular Patterns (Cambridge Univ. Press,, Cambridge, 1991).

[16] A. Crisanti, M. Falcioni, G. Paladin and A. Vulpiani, Riv. Nuovo Cimento 14, 1 (1991) 
[17] C. Marchioro and M. Pulvirenti, Mathematical theory of uncompressible nonviscous fluids, Applied mathematical science, Vol. 96 (SpringerVerlag, New York, 1994).

[18] E. A. Novikov and Y. B. Sedov, Sov. Phys. JETP 48, 440 (1978)

[19] H. Aref and N. Pomphrey, Phys. Lett. A 78, 297 (1980).

[20] X. Leoncini, L. Kuznetsov and G. M. Zaslavsky, Phys. Rev. E 63, p. 036224 (2001).

[21] Flandrin P., Temps-Fréquence, Hermès, 1993-1998.

[22] Almeida L. B., IEEE Trans. Sig. Processing 42 (11), 3084-3091 (1994).

[23] F. Clairet, B. Ricaud, F. Briolle, S. Heuraux, C. Bottereau, Rev. Sci. Instrum. 82, 8 (2011), 9p.

[24] Mallat S., Zhang Z., IEEE Trans. Sig. Processing, 41(12), 3397-415 (1993). 